\title{
Diffractive vector meson leptoproduction and spin effects
}

\section{S.V. Goloskokov*}

Bogoliubov Laboratory of Theoretical Physics, Joint Institute for Nuclear Research, Dubna 141980, Moscow region, Russia

E-mail: goloskkv@theor.jinr.ru

\begin{abstract}
We analyse spin effects in diffractive vector meson leptoproduction at small $x$ on the basis of the generalized parton distribution (GPD) approach. We take into account quark transverse degrees of freedom in the hard subprocess. We calculate amplitudes for the longitudinally and transversely polarized photons and vector mesons. Our results on the cross section and spin density matrix elements (SDME) are in fair agreement with the DESY experiments. Predictions for HERMES and COMPASS energy range are made. The predicted double spin longitudinal $A_{L L}$ asymmetry is not small at HERMES energies.
\end{abstract}

DIFFRACTION 2006 - International Workshop on Diffraction in High-Energy Physics September 5-10 2006

Adamantas, Milos island, Greece

${ }^{*}$ Speaker. 


\section{Introduction}

In this report, we study spin effects in vector meson leptoproduction at large energies within the GPD approach on the basis of [1]. At small $x$-Bjorken $(x)$ the process factorizes [2] into a hard meson leptoproduction off gluons and GPD (Fig. 1).

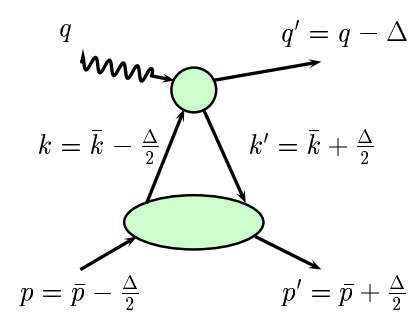

Figure 1: The handbag diagram for the meson electroproduction off proton.

Unfortunately, it is difficult to study spin effects in vector meson leptoproduction in the generally used standard collinear approximation (SCA). Only the amplitude with longitudinally polarized virtual photon and vector meson (LL) amplitude can be calculated in the SCA. To analyse spin effects, one should have information about ampitudes with transversally polarized photons. The TT and TL transition amplitudes where the symbols $L$ and $T$ refer to longitudinally and transversely polarized photons and vector mesons, respectively, exhibit in the SCA the infrared singularities [3] which breakdown factorization.

Our model is based on the modified perturbative approach (MPA) [4] which includes the quark transverse degrees of freedom accompanied by Sudakov suppressions. The transverse quark momentum regularizes the end-point singularities in the TT and TL amplitudes. This gives a possibility to calculate these amplitudes in the MPA and suppress a contribution from the end-point region in the LL amplitude. As a result, we obtain a reasonable description of the $\mathrm{H} 1$ and ZEUS data [5, 6] for electroproduced $\rho$ and $\phi$ mesons at small $x[1,7]$.

In this report, we analyse the $\phi$ meson leptoproduction. Within the MPA we calculate the three transition $L L, T T$ and $T L$ amplitudes. We present our results for the cross sections and SDME at DESY energies and give some predictions for the HERMES and COMPASS energy range.

\section{Leptoproduction of Vector Mesons in the GPD approach}

The leading-twist wave function describes the longitudinally polarized vector mesons. To study spin effects, we should consider the higher order wave function which describes transversely polarized mesons. Here we use the $k$ - dependent wave function proposed in [8].

The gluon contribution to the leptoproduction amplitudes is predominant in the low- $x$ region. For $t \sim 0$ and positive proton helicity the amplitude reads as a convolution of the hard subprocess amplitude $\mathscr{H}^{V}$ and a large distance unpolarized $H^{g}$ and polarized $\widetilde{H}^{g}$ gluon GPDs (Fig. 1):

$$
\begin{aligned}
\mathscr{M}_{\mu^{\prime}+, \mu+} & =\frac{e}{2} \mathscr{C}_{V} \int_{0}^{1} \frac{d \bar{x}}{(\bar{x}+\xi)(\bar{x}-\xi+i \varepsilon)} \\
& \times\left\{\left[\mathscr{H}_{\mu^{\prime}+\mu+}^{V}+\mathscr{H}_{\mu^{\prime}-\mu-}^{V}\right] H^{g}(\bar{x}, \xi, t)\right. \\
& \left.+\left[\mathscr{H}_{\mu^{\prime}+, \mu+}^{V}-\mathscr{H}_{\mu^{\prime}-, \mu-}^{V}\right] \widetilde{H}^{g}(\bar{x}, \xi, t)\right\} .
\end{aligned}
$$


Here $\mu\left(\mu^{\prime}\right)$ denotes the helicity of the photon (meson), $\bar{x}$ is the momentum fraction of the transversally polarized gluons, and the skewness $\xi$ is related to Bjorken- $x$ by $\xi \simeq x / 2$. The flavor factors are $C_{\rho}=1 / \sqrt{2}$ and $C_{\phi}=-1 / 3$. The polarized $\widetilde{H}^{g}$ GPD at small $\bar{x}$ is much smaller than the GPD $H^{g}$ and will be important in the $A_{L L}$ asymmetry only.

The subprocess amplitude $\mathscr{H}^{V}$ is represented as the contraction of the hard part $F$, which is calculated perturbatively, and the non-perturbative meson wave function $\phi_{V}$

$$
\mathscr{H}_{\mu^{\prime}+, \mu+}^{V} \pm \mathscr{H}_{\mu^{\prime}-, \mu-}^{V}=\frac{2 \pi \alpha_{s}\left(\mu_{R}\right)}{\sqrt{2 N_{c}}} \int_{0}^{1} d \tau \int \frac{d^{2} \mathbf{k}_{\perp}}{16 \pi^{3}} \phi_{V}\left(\tau, k_{\perp}^{2}\right) F_{\mu^{\prime} \mu}^{ \pm}
$$

The wave function is chosen in the Gaussian form

$$
\phi_{V}\left(\mathbf{k}_{\perp}, \tau\right)=8 \pi^{2} \sqrt{2 N_{c}} f_{V} a_{V}^{2} \exp \left[-a_{V}^{2} \frac{\mathbf{k}_{\perp}^{2}}{\tau \bar{\tau}}\right] .
$$

Here $\bar{\tau}=1-\tau, f_{V}$ is the decay coupling constant and the $a_{V}$ parameter determines the value of average transverse momentum of the quark in the vector meson. Generally, values of $f_{V}, a_{V}$ are different for longitudinal and transverse polarization of the meson.

In calculation of the subprocess amplitude within the MPA [4] we keep the $k_{\perp}^{2}$ terms in the denominators of the amplitudes. We keep the $k_{\perp}^{2}$ terms in the numerator of the TT amplitude too. The gluonic corrections are treated in the form of the Sudakov factors which additionally suppress the end-point integration regions. In the model the following hierarchy of the amplitudes is obtained

$$
\mathrm{LL}: \quad \mathscr{M}_{0 v, 0 v}^{V(g)} \propto 1 ; \quad \mathrm{TL}: \quad \mathscr{M}_{0 v,+v}^{V(g)} \propto \frac{\sqrt{-t}}{Q} ; \quad \mathrm{TT}: \quad \mathscr{M}_{+v,+v}^{V(g)} \propto \frac{\mathbf{k}_{\perp}^{2}}{Q M_{V}} .
$$

The $L \rightarrow T$ and $T \rightarrow-T$ transition amplitudes are small and neglected in our analysis.

The GPD is a complicated function which depends on three variables. For a small momentum transfer $t \sim 0$ we use the double distribution form [9]

$$
H^{g}(\bar{x}, \xi, t)=\left[\Theta(0 \leq \bar{x} \leq \xi) \int_{\frac{\bar{x}-\xi}{1+\xi}}^{\frac{\bar{x}+\xi}{1+\xi}} d \beta+\Theta(\xi \leq \bar{x} \leq 1) \int_{\frac{\bar{x}-\xi}{1-\xi}}^{\frac{\bar{x}+\xi}{1+\xi}} d \beta\right] \frac{\beta}{\xi} f\left(\beta, \alpha=\frac{\bar{x}-\beta}{\xi}, t\right)
$$

with the simple factorizing ansatz for the double distributions $f(\beta, \alpha, t)$

$$
f(\beta, \alpha, t \simeq 0)=g(\beta) \frac{3}{4} \frac{\left[(1-|\beta|)^{2}-\alpha^{2}\right]}{(1-|\beta|)^{3}} .
$$

The ordinary gluon distribution is parameterized as

$$
\beta g(\beta)=\beta^{-\delta\left(Q^{2}\right)}(1-\beta)^{5} \sum_{i=0}^{2} c_{i} \beta^{i / 2} .
$$

The parameter $\delta\left(Q^{2}\right)$

$$
\delta\left(Q^{2}\right)=0.17+0.07 \ln \left(Q^{2} / Q_{0}^{2}\right)-0.005 \ln ^{2}\left(Q^{2} / Q_{0}^{2}\right)
$$

determines the behavior of the gluon distribution at low $x$ and the energy dependence of the cross section at high energies. Other parameters in (2.7) are taken from comparison with the CTEQ5M parameterization [10].

This model gives a possibility to calculate GPD [1] through the gluon distribution $g(\beta)$. For the three $\xi$ values the gluon GPD is shown in Fig. 2. 


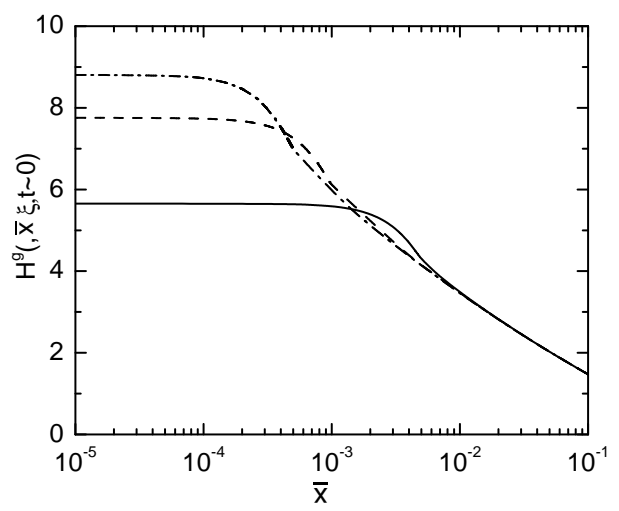

Figure 2: Model results for the GPD $H^{g}$ in the small $\bar{x}$ range. The solid (dashed, dash-dotted) line represents the GPD at $\xi=5(1,0.5) \cdot 10^{-3}$

\section{Cross section and spin observables}

Experimentally, only the slope of the $\gamma^{*} p \rightarrow V p$ cross section is measured. The $t$ - dependence of the amplitudes is generally unknown. For simplicity, we parameterize it in the exponential form $M_{i i}(t)=M_{i i}(0) e^{t B_{i i} / 2}$ for $L L, T T, T L$ transitions and suppose that $B_{L L} \sim B_{T T} \sim B_{T L}$.

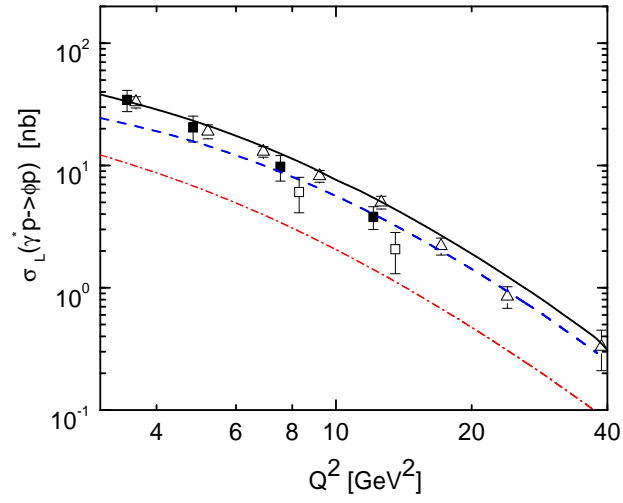

(a)

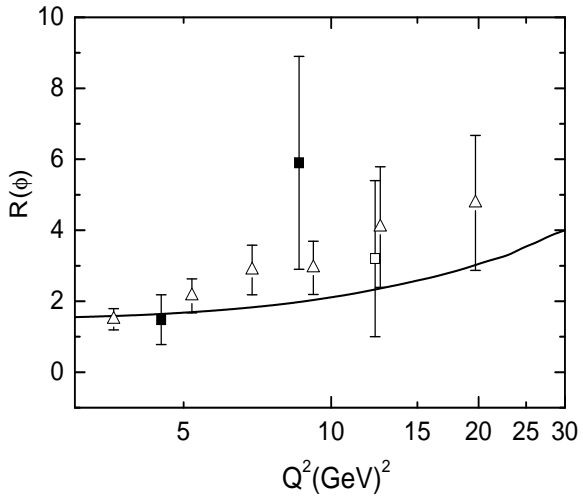

(b)

Figure 3: (a) Longitudinal cross sections of $\phi$ production at $W=75 \mathrm{GeV}$. Full line cross section, dashedgluon contribution, dashed-dot - gluon-strange interference. (b) The ratio of longitudinal and transverse cross sections for the $\phi$ production at $W=75 \mathrm{GeV}$ and $t=-0.15 \mathrm{GeV}^{2}$. Data are from $\mathrm{H} 1$ and ZEUS.

The longitudinal cross section for the $\phi$ production integrated over $t$ is shown in Fig. 3a. Estimations for the vector meson production are obtained using $f_{\phi L}=.237 \mathrm{GeV}, a_{\phi L}=0.45 \mathrm{GeV}^{-1}$; $f_{\phi T}=.190 \mathrm{GeV} ; a_{\phi T}=0.6 \mathrm{GeV}^{-1}$. In addition to the gluon distribution the gluon-sea-quark interference is shown in Fig. 3a. The typical contribution of the interference to $\sigma_{L}$ does not exceed 40 $\%$ with respect to the gluon one. Thus, the gluon term really gives the predominant contribution to the cross section and we find good agreement of our results with H1 and ZEUS experiments [5] at HERA. Note that the uncertainties in the gluon GPD provide errors in the cross section about $25-35 \%$. This is the same order of magnitude as the gluon-sea interference. The model results 
for the ratio of the cross section with longitudinal and transverse photon polarization $R$ for the $\phi$ production are shown in Fig. $3 \mathrm{~b}$ and are consistent with the H1 and ZEUS experiments $[5,6]$.
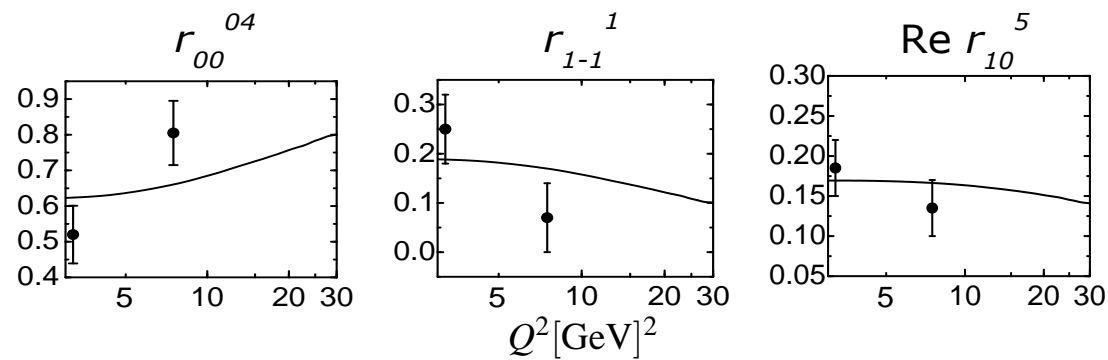

Figure 4: The $Q^{2}$ dependence of SDME on the $\phi$ production at $t=-.15 \mathrm{GeV}^{2}$ and $W=75 \mathrm{GeV}$. Data are taken from $\mathrm{H} 1$.

SDME are determined in terms of interference of different amplitudes of vector meson photoproduction. Thus, they are sensitive to the LL,TT, TL amplitude structure and to the vector meson wave function. In Fig. 4, we present our results for the three SDME in the DESY energy range. Description of experimental data [5] is reasonable. Our results for other SDME can be found in [1]. We can conclude that our amplitude hierarchy (2.4) is supported by experiment.

Let us discuss the energy dependence of the cross section. At small $x$ they behave as

$$
\sigma_{L} \propto W^{4 \delta\left(Q^{2}\right)} .
$$

In the Regge picture the power $\delta$ (2.8) is associated with the Pomeron intercept $\alpha_{P}(0)=1+\delta\left(Q^{2}\right)$. In Fig. 5, we show our results for the $\phi$ - production cross section. The data [6] in Fig. 5 are recalculated to the longitudinal cross section using the ratio $R=1.5$ at $Q^{2}=3.8 \mathrm{GeV}^{2}$ [6]. One can see that the preliminary HERMES [11] and HERA data [5, 6] are described well.

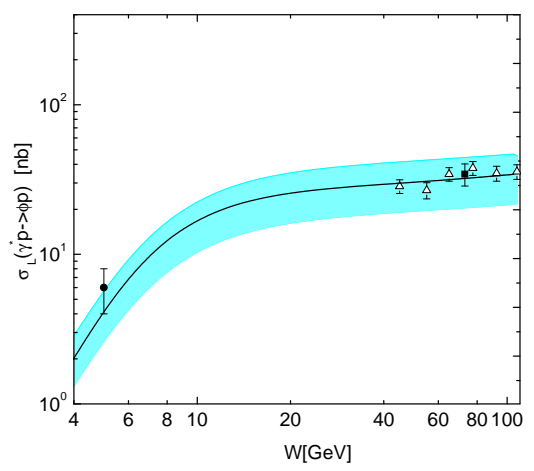

Figure 5: The longitudinal cross section for $\phi$ production vs. $W$ for $Q^{2}=3.8 \mathrm{GeV}^{2}$.

Thus, we can make some predictions for $\phi$ production SDME at intermediate energies. Our results for COMPASS are shown in Fig. 6. It can be seen that the energy dependencies of SDME are weak.

The $A_{L L}$ asymmetry for a longitudinally polarized beam and target is sensitive to the polarized GPD. Really, the leading term in the $A_{L L}$ asymmetry integrated over the azimuthal angle is determined through the interference between the unpolarized GPD $H^{g}$ and polarized $\widetilde{H}^{g}$ distributions. 

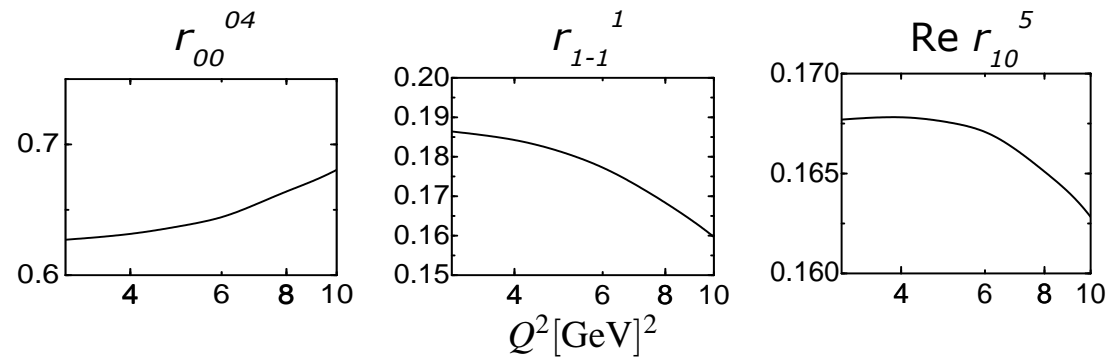

Figure 6: The predicted $Q^{2}$ dependence of SDME on the $\phi$ production at $t=-.15 \mathrm{GeV}^{2}$ and the COMPASS energy $W=10 \mathrm{GeV}$.

We expect a small value for the $A_{L L}$ asymmetry at high energies because it is of the order of the ratio $\left\langle\widetilde{H}^{g}\right\rangle /\left\langle H^{g}\right\rangle$ which is small at low $x$. At COMPASS energies $W=10 \mathrm{GeV}$ the gluon

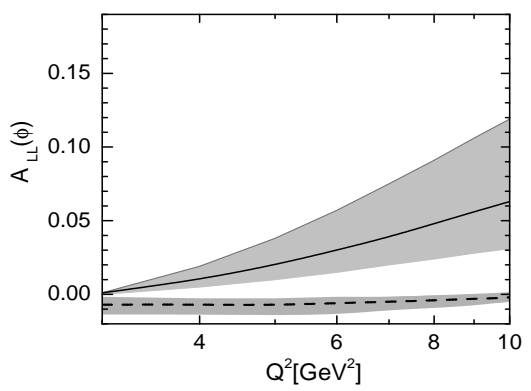

Figure 7: The $A_{L L}$ asymmetry of $\phi$ meson production at $W=5 \mathrm{GeV}$ (solid line) and $W=10 \mathrm{GeV}$ (dashed line); $y \simeq 0.6$. The shaded bands reflect the uncertainties in $A_{L L}$ due to the error in $\widetilde{H}^{g} \propto \Delta g$.

contribution to the $A_{L L}$ asymmetry of vector meson production is small (Fig. 7). At HERMES energies $W=5 \mathrm{GeV}$ the major contribution to the amplitudes comes from the region $0.1 \leq \bar{x} \leq 0.2$ where $\Delta g / g$ is not small, which leads to a large value of the $A_{L L}$. The asymmetry is shown in Fig. 7 together with the uncertainties in our predictions due to the error in the polarized gluon distribution. Note that our predictions should be valid for the $\phi$ production. In case of $\rho$ production the polarized quark GPD should be essential in the $A_{L L}$ asymmetry in the low energy range.

\section{Conclusion or Summary}

We have analyzed electroproduction of $\phi$ meson at small Bjorken- $x$ in the handbag approach where the process amplitudes are factorized into the gluonic generalized parton distributions and a partonic subprocess, $\gamma g \rightarrow(q \bar{q}) g, q \bar{q} \rightarrow V$. The subprocess was calculated [1] within the modified perturbative approach where the transverse momenta of the quark and antiquark as well as Sudakov corrections were taken into account. These effects suppress the contributions from the end-point regions where one of the partons entering the meson wave function becomes soft and factorization breaks down in the collinear approximation. This allowed us to investigate the amplitudes for all kinds of transitions from longitudinally and transversally polarized virtual photons to polarized vector mesons. This give a possibility to study spin effects in vector meson production. Within the GPD approach we find fine description of the $Q^{2}$ dependence of the cross section, $R$ ratio and 
SDME for the $\rho$ meson production at HERA energies. More results for $\rho$ and $\phi$ production can be found in [1]. Some comparison with the two-gluon exchange model [12] can be found in [1] too.

We would like to point out that study of SDME gives an important information about different $\gamma \rightarrow V$ hard amplitudes. By analyses of $t$ dependences of SDMEs the diffraction peak slopes of the $T T$ and $T L$ amplitudes can be defined. Unfortunately, the data on spin observables have large experimental errors. The new experimental results with reduced errors in SDME at $Q^{2} \geq 3 \mathrm{GeV}^{2}$ are extremely important. Information about polarized gluon distribution can be obtained from the $A_{L L}$ asymmetry on $\phi$ meson production which is sensitive to $\Delta G$. Thus, we can conclude that the vector meson photoproduction at small $x$ is an excellent tool to study the gluon GPD.

This work is supported in part by the Russian Foundation for Basic Research, Grant 06-0216215 and by the Heisenberg-Landau program.

\section{References}

[1] S.V. Goloskokov, P. Kroll, Vector meson electroproduction at small Bjorken-x and generalized parton distributions, Euro. Phys. J. C42 (2005) 281 [hep-ph/0511307].

[2] X. Ji, Deeply Virtual Compton Scattering, Phys. Rev. D55 (1997) 7114 [hep-ph/ 9609381$]$; A.V. Radyushkin, Scaling Limit of Deeply Virtual Compton Scattering, Phys. Lett. B380 (1996) 417 [hep-ph/9604317].

[3] L. Mankiewicz and G. Piller, Comments on Exclusive Electroproduction of Transversely Polarized Vector Mesons, Phys. Rev. D61 (2000) 074013 [hep-ph/9905287]; I.V. Anikin and O.V. Teryaev, Genuine twist 3 in exclusive electroproduction of transversely polarized vector mesons, Phys. Lett. B554 (2003) 51 [hep-ph/ 0211028].

[4] J. Botts, G. Sterman, Hard Elastic Scattering In QCD: Leading Behavior, Nucl. Phys. B325 (1989) 62.

[5] C. Adloff et al. [H1 Collaboration], Measurement of elastic electroproduction of $\phi$ mesons at HERA, Phys.Lett.. B483 (2000) 360 [hep-ex / 0005010 ].

[6] S. Chekanov et al. [ZEUS Collaboration], Exclusive electroproduction of $\phi$ mesons at HERA, Nucl.Phys. B718 (2005) 3 [hep-ex/ 0504010$].$

[7] S. Goloskokov, Deeply virtual electro-production of vector mesons and spin effects, to appear in Proc. of SPIN-Praha-2006 conference, Czech. J. Phys. 56 (2006) [hep-ph/ 0610049 ]

[8] J. Bolz, J.G. Körner and P. Kroll, $l=0$ to $l=1$ transition form-factors, Z. Phys. A350 (1994) 145 [hep-ph/9403319].

[9] I. V. Musatov and A. V. Radyushkin, Evolution and models for skewed parton distributions, Phys. Rev. D61 (2000) 074027 [hep-ph/ 9905376$].$

[10] J. Pumplin, D. R. Stump, J. Huston, H. L. Lai, P. Nadolsky and W. K. Tung, New generation of parton distributions with uncertainties from global QCD analysis, JHEP 0207 (2002) 012

[hep-ph/0201195].

[11] N. Bianchi, Exclusive Meson and Photon Production at HERMES, Int.J. Mod. Phys. A.18 (2003) 1311.

[12] S. J. Brodsky et al., Diffractive leptoproduction of vector mesons in QCD, Phys. Rev. D 50 (1994) 3134 [hep-ph/9402283]. 